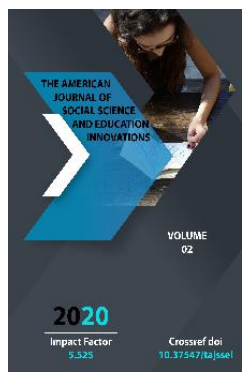

\title{
Methods Of Diagnosing A State Of Depression In A Person's Behavior
}

Journal Website: http://usajournalshub.c om/index,php/tajssei

\section{ABSTRACT}

The article discusses the ways in which emotional stress in the learning process can be manifested at different levels, strengths and qualities, as well as methods to study experiences such as depression, depression, low self-esteem in a person as an emotional state information is given.

\section{KEYWORDS}

Stress, frustration, anxiety, aggression, introspection, misbehavior, coping strategy.

\section{INTRODUCTION}

Relevance of the topic. Factors influencing the achievement of business goals have both positive and negative qualities. Emotional stress is one of the most common causes of stress. Problems related to a person's emotional states and their place in various activities and relationships have been studied extensively in psychology.

P. Lazarus, an American psychologist who has studied the problem of emotional states, proposes to distinguish between the concepts of physiological and psychological stress in order to fully understand the essence of the concepts of emotional tension and stress. According to him, these cases differ from each other depending on the nature of the causative agent, the mechanism of its occurrence and the type of response.

Emotional tensions in the learning process can manifest at different levels, strengths, and qualities. These include stress, frustration, anxiety, aggression, depression, and more. Depression is the emotional state of a person that is manifested in such experiences as depression, depression, and low self-esteem, 
and at the same time can have a negative impact on a person's development.

During depression, a person's self-confidence decreases, which in turn can negatively affect his ability to set big goals and enter into interpersonal relationships. This can be even more dangerous, especially during adolescence, and can play an important role in the formation of his personality, the composition of individual characteristics. The aim of the study was to determine the factors that cause depression in adolescents and their impact on the formation of adolescence.

Today, the study of the nature of emotional stress, as well as the nature of depression, the study of its manifestations, and their prevention has risen to the level of a more pressing problem.

\section{THE MAIN PART}

Several scientists have studied the effects of depression. There are objective and subjective methods of assessing the level of depression. Objective methods include assessment of the cardiovascular and respiratory systems, assessment of the activity of the autonomic nervous system, assessment of the body's psychomotor reactions during depression, and other methods.

The most commonly used physiological methods for assessing the condition of the cardiovascular and respiratory systems are recording the frequency of heartbeats; determination of blood pressure readings; electrocardiography; is to determine the depth and frequency of breathing.

In recent years, the use of complex methods of studying depression and stress has become increasingly popular. This is because the body responds in a complex and systematic way to the effects of stress. Therefore, the study of the interactions of the nervous, humoral, cardiovascular and muscular systems is of great interest.

The views expressed by scientists from the Bulgarian Institute of Hygiene are noteworthy. According to him, isolated reactions provide less information than complex established reactions. One of the simplest and quickest ways to assess functional status during stress is to measure the heart rate.

Studies have shown that; during exams, the pulse in freshmen ranged from 79 to 98 beats per minute. Heart rate during the exam is different from normal. During the exams, the pulse reached $120-150$ in $3.8 \%$ of students, and even tachycardia and bradycardia were observed in some students. The effects of the sympathetic and parasympathetic nervous systems are particularly pronounced in students with a weak nervous system, and the stress is very severe.

Research has shown that the degree of depression depends not only on the strength of the factors that cause it, but also on the type of higher nervous activity of the person who feels it.

Subjective methods of assessing the level of depression include psychological (sheet and projective) tests, introspection, selfmonitoring of emotional state, and others.

In modern psychology, many tests are used to study the different components of stress and other depressions. Some tests examine the subjective components of anxiety, while others examine their vegetative manifestations.

The use of sheet tests may not provide a complete analysis of the causes of depression. In this case, the use of the method of introspection, that is, self-analysis in determining the causes of stress, as it continues, provides more information.

1. At the first sign of emotional stress, it is helpful to ask the following standard questions. 
Am I overwhelmed by negative emotions?

What am I thinking about now?

How are my muscles? Are they

overstressed?

How do I breathe?

2. In stress analysis, one should not only focus on the problem, but also be able to analyze the situation in depth. It is necessary to pay attention to the possibilities of its development, the qualities of its action in times of stress, the consequences that occur after the completion of the main actions.

3. A depressive condition is an event or phenomenon that can trigger stressrelated reactions. To find out, ask yourself the following questions. What contributed to the development of depression? When did this happen? What did I do? Who was with me? What were my thoughts before the tension started?

4. To find out how stress is going on, you need to ask the following questions. How did you feel during the stress? What physiological sensations do you experience during stress? What was I thinking at the time? What thoughts were in my mind?

GM Granovskaya and IM Nikolskaya note that the concept of coping is close in its meaning to the concept of psychological protection. Coping behavior occurs when a person is in a state of crisis. Any crisis situation presupposes the existence of a certain attitude, depending on the objective conditions and the level of importance of the person to it. This connection is accompanied by the emotional response of different behaviors and the level of intensity. The main characteristics of a crisis situation are stress, inner experiences in overcoming life events and traumas, feelings of humiliation, and the need for external spiritual support. There are different classifications of coping strategies. Some theories of coping behavior include the following basic strategies:

1. Troubleshooting;

2. Seeking social assistance;

3. Pull yourself aside.

In addition, the coping strategy takes place in three areas.

1. Behavioral circle;

2. Cognitive circle;

3. Emotional circle.

During adolescence, the process of learning how to cope with life's challenges continues. The role of adults, who are always supportive, is invaluable.

Teen coping behaviors have not yet been studied. The main purpose of the study was to study the types of behaviors of adolescents in relation to the content of the stress-related socio-psychological situation. The nature and types of onset of depression depend on the nature of the socio-psychological situation. The study focuses on the following issues: to determine the extent and characteristics of adolescents' exposure to stress, in which sphere, to determine the strategy for coping with stress in adolescents and young adults, the current situation in the country. 'Assessing the potential manifestations of the sociopsychological adaptation of smiles.

Based on the research of EN Tumanova and taking into account the specific crisis situations in the lives of adolescents, a questionnaire "Coping strategy in crisis situations" was developed for adolescents. Adolescents are asked to identify the types of reactions to the following life events. These are: family (family quarrels), relationships with friends (disagreements with friends), relationships with adults (disagreements with the teacher), learning activities (failure in written work), health (illness, trauma ). In order to assess the form of vulnerability, 
considerations related to this or that coping strategy are offered.

A. Behavioral Coping Strategies:

- $\quad$ Active overcoming, altruism;

- $\quad$ Partially adaptive;

- $\quad$ Seeking social assistance;

- Distraction

- $\quad$ Noadaptiv;

- $\quad$ Not joining anyone;

- $\quad$ Compensation for doping (or drug) use;

- $\quad$ Leaving the real situation.

B. Cognitive coping strategy:

- Adaptive;

- $\quad$ Search for a solution to the problem;

- $\quad$ Learn content;

- $\quad$ Partially adaptive;

- $\quad$ Relying on the opinions of other loved ones;

- Rejection;

- $\quad$ Avoiding the problem;

- $\quad$ Feeling we have 'Run out of gas' emotionally.

C. Emotional coping strategy:

- $\quad$ Adaptive;

- $\quad$ Consciousness;

- $\quad$ Partially adaptive;

- $\quad$ Seek emotional help;

- $\quad$ Emotional control;

- $\quad$ Feelings of guilt;

- Obedience.

The norms of the adaptive strategy of the group of teenagers and young adults to overcome the crisis will not change. The nonadaptive form of susceptibility may decrease with age in order to be able to implement a partially adaptive coping strategy. During adolescence, there are non-adaptive forms of stress management. Particularly adaptive forms of sensitivity are observed during early adolescence in adulthood. In adolescents, as well as in adolescents, the rate of adaptive stress management is largely determined by the size of the family relationship. As a result, adolescents are more likely to adapt to difficult family situations.

As adaptation to the difficult life situations of adulthood increases, there is an increase in health impairment in problematic situations during early adolescence, and a decrease in adaptive strategies for coping with stress in adult adolescents. It depends on the selfdetermination of the situation, the value of health in the process of solving the problems of personal independence. Physical trauma or a painful illness can seem unbearable to a teenager and can destroy a great deal of mental strength. Thus, foreign psychologists have conducted theoretical and empirical studies of the manifestations and course of depression in adolescents. These studies are of great importance today.

Thus, it is possible to diagnose the tensions that arise in a person's behavior, in the process of relationships with others, and under the influence of socio-economic conditions, and to monitor the dynamics of his development.

Depression is a change in the adolescent's psyche that results in nervousness, fear, anxiety, depression, depression, low selfesteem, and, in the most severe cases, suicide. cases are also observed.

As a result, it is important to identify changes in students during adolescence, to prevent depression and its consequences, to work with adolescents who are prone to depression, and in this case, to understand their identity, self-awareness. The formation of a well-rounded person is the most pressing issue for this period. One of the most pressing issues in this study was the study of the predisposition to depression in adolescents.

In the first phase of the study, its goal was to diagnose the predisposition and manifestations of depression in adolescents and adolescents, emotional stress in adolescents - with the state of depression and 
their personality traits and self-assessment. important goals were identified, such as studying addiction, teaching depression and ways out of it, and finding ways to prevent depression. The study participants were selected and briefed in accordance with the guidelines.

The depression study scale is adapted by $\mathrm{TI}$ Balashova and lasts for 20-30 minutes. Students will be given the following instructions: Without thinking long and hard about the following 20 questions, choose one of the answers: "Never", "Rarely", "Sometimes", “Often", "Almost always". mark."

In the second phase of the survey (data collection), the respondents were given a questionnaire and the text of the questionnaire, and the answers were collected. Students answered 20 questions.

The key to the methodology was used in the third phase, which consisted of analyzing the research results.

The degree of depression is determined using the following formula:

$D \cdot D=W$ right $+W$ inverse

W correct $=1,2,3,4,7,8,9,10,13,15,19$

W reverse $=2,5,6,11,12,14,16,17,18,20$

If the result is less than 50 points - there is no I-depression:

50-59 points - II-degree depression

Up to 60-69 points - III subdepression, ie masked

Up to 70-80 points - IV- represents true depression.

During the study, the respondents' answer sheets were quantified using the method key. Qualitative analysis of the results shows that the average score of the group was 43.1 points. This suggests that almost all of those surveyed did not have depression. $26.6 \%$ of the subjects had mild depression and $74.4 \%$ had no depression at all.
A self-assessment method was used to determine the relationship between adolescent depression and self-esteem.

Words that express certain personality traits to the examinee, such as care, carelessness, thoughtfulness, aggression, intelligence, pride, rudeness, cheerfulness, caring, jealousy, shyness, sincerity, inquisitiveness whimsy, principled, poetic, invisible, open-faced, sarcastic, foolish, bold, self-absorbed in work, restraint, sadness, honesty, endurance, cowardice, curiosity, perseverance, before the Examiner He finds among them words that express the qualities he possesses. Then find the words you want to have. 50 sets of words were suggested, such as fun, carelessness, initiative.

The examiners carefully review the given words and then write them in two lines (10-20 words per line). In the first line he writes the words that correspond to his ideal, and in the second line he writes the words that he does not have. The second line can be conditionally called 'anti-ideal'. The words in the first line make up the "positive" plural, and the words in the second line make up the "negative" plural.

They are the criteria.

The test taker then finds the words that match the attributes he or she has among the "positive" and "negative" plurals. In doing so, the test taker should consider whether or not these qualities are present ("Yes" or "No").

The analysis of the methodology results is performed as follows. The number of adjectives chosen by the test taker is counted for each plural and divided by the total number of words in the corresponding criterion. If the coefficients on the positive plural are close together, it means that the test taker overestimated himself and was not critical of himself. The fact that the coefficient of the negative set is close to zero is an understatement. A coefficient close to 0.5 
indicates that the test taker has a normal, average self-esteem. At the same time, he shows that he is self-critical, without overestimating himself.

Among those surveyed, there were those with low, medium, and high self-esteem.

\section{CONCLUSION}

A comparative analysis of the data showed that adolescents with low self-esteem may be more prone to depression. Adolescents who value their abilities and other characteristics are less likely to develop this tendency. Depression does not occur in adolescents who have a variety of life challenges, interpersonal problems, self-control in failures and failures, and perseverance in achieving their goals. Frequent recurrences of failures and setbacks in activities can create the most favorable conditions for a depressive condition to occur. Depression can also be caused by a lack of self-esteem.

With the help of psychodiagnostic methods and techniques, the timely detection of depressive disorders and the development of appropriate practical measures;

Gradual depression can lead to a variety of mental disorders, neurosis, and even suicidal ideation.

\section{REFERENCES}

1. Bodopyanova N.Ye. Psychodiagnostics of stress. Peter Press. 2009. 336 p.

2. Gamezo M.V., Domashenko I.A. Atlas in psychology. M. 1986

3. Lowen A. Depression and the Body $M$. 1968

4. Naenko N.I. Mental tension M. 1996

5. Nemov RS, Psychology. B-3x book., Book1, M. 1998

6. Nemchin E.I. The state of neuropsychic tension M. 1993.
7. Nishonova Z.T. Qurbonova Z. Psychodiagnostics. T. 2008.

8. Rusalov V.M., Biological bases of individual psychological differences, $M$. Nauka, 1991

9. Shcherbakov Al, Practicum in Youth Psychology and Pedagogical Psychology. T. 1991

10. Sherbatikh Y.D. Psychology of stress and ego correction methods. M. 2006 